\title{
ASPECTOS DO MANEJO DE RESÍDUOS QUÍMICOS EM INSTITUIÇÃO DO ENSINO SUPERIOR
}

Tatiane Bonametti Veiga ${ }^{1}$

Sílvia Carla da Silva André ${ }^{2}$

Angela Maria Magosso Takayanagui ${ }^{3}$

RESUMO: Os resíduos químicos tornaram-se alvo de grande preocupação devido sua diversidade, composição e potencial de periculosidade. Este trabalho tem como objetivo verificar as diferentes fases do manejo de resíduos químicos nos laboratórios e serviços no Campus da Universidade de São Paulo em Ribeirão Preto. O levantamento foi realizado a partir da aplicação de um questionário junto aos responsáveis pelos laboratórios e serviços desse Campus, com a participação de 199 sujeitos, respondendo por um percentual de $66,6 \%$ dos locais da pesquisa. Os dados revelaram que em $80,9 \%$ dos locais participantes da pesquisa havia a geração de resíduos químicos. Em relação às fases de manejo interno (segregação, acondicionamento, identificação, armazenamento, coleta, transporte e tratamento interno) foi verificado que procedimentos como a segregação na fonte, o acondicionamento em recipientes compatíveis com o tipo de resíduo, a identificação das embalagens, encontravam-se, em sua maioria, adequados à legislação brasileira. Segundo os sujeitos, $64,3 \%$ desses locais não realizavam tratamento interno, a coleta interna era realizada, em sua maioria $(87,5 \%)$, por funcionários da instituição ou terceirizado e o transporte interno era substancialmente manual $(63,3 \%)$. O aumento do desconhecimento foi observado nas fases de manejo externo (coleta, transporte, tratamento e disposição final), identificando-se conceitos errôneos nas respostas dos sujeitos. A partir da realização deste estudo, observa-se a importância da elaboração de

\footnotetext{
${ }^{1}$ Doutoranda em Ciências, Programa de Pós-graduação em Enfermagem em Saúde Pública, Universidade de São Paulo - USP. Engenheira Civil. tati.veiga@yahoo.com.br.

${ }^{2}$ Doutoranda em Ciências, Programa de Pós-graduação em Enfermagem em Saúde Pública, Universidade de São Paulo - USP. Enfermeira. silviacarlabjp@gmail.com.br.

${ }^{3}$ Doutora em Enfermagem. Universidade de São Paulo - USP. Professora Associada da Escola de Enfermagem de Ribeirão Preto (EERP/USP). amtakay@eerp.usp.br.
} 
Plano de Gerenciamento de Resíduos para as Instituições de Ensino Superior, destacando também a relevância da aplicação de um programa de educação em serviço, de forma permanente, a fim de contribuir para o gerenciamento ambiental nessas instituições.

Palavras-chave: Gerenciamento de Resíduos. Instituição de Ensino Superior. Resíduos Químicos.

\section{INTRODUÇÃO}

O histórico da humanidade mostra a evolução do homem no ponto de vista biológico, social, cultural e tecnológico, e reflete as interfaces de sua estreita relação com a natureza (PUGLIESI; GIL; SCHALCH, 2009). Essa evolução trouxe muitos benefícios e comodidade à sociedade, mas também fez despontar muitos problemas, com sérios danos ao ambiente e à saúde humana.

Uma das grandes preocupações no mundo moderno corresponde ao aumento da quantidade de resíduos gerada diariamente, situação que se agrava com o acréscimo do consumo de materiais descartáveis (BUENO; LEAL, 2012). Entre os diferentes tipos de resíduos produzidos no espaço urbano, encontram-se os resíduos de serviços de saúde (RSS), que, mesmo representando uma pequena quantidade dos resíduos, despertam a preocupação por apresentarem um risco potencial, sendo fonte de substâncias perigosas (TAKAYANAGUI, 2005). Esses resíduos quando não manejados de forma adequada podem provocar a disseminação de doenças e a contaminação dos recursos naturais como solo, água e ar.

Pela NBR 10.004/04, a periculosidade dos resíduos está relacionada às suas propriedades físicas, químicas ou infectocontagiosas que apresentem risco à saúde e ao ambiente, a saber: Risco à saúde que corresponde à probabilidade da ocorrência de efeitos adversos à saúde devido à exposição humana a agentes físicos, químicos ou biológicos, que podem provocar aumento da mortalidade ou incidência de doenças, dentro de um período determinado de tempo ou idade; Risco ao meio ambiente referente à probabilidade da ocorrência de efeitos adversos ao ambiente decorrentes à ação dos agentes físicos, químicos ou biológicos que podem causar condições ambientais potencialmente perigosas (ABNT, 2004; BRASIL, 2006). 
$\mathrm{Na}$ Norma Regulamentadora $\mathrm{n} \cong$ 9, os riscos ambientais são considerados os agentes físicos, químicos e biológicos presentes nos ambientes de trabalho, que podem causar danos à saúde, devido a sua concentração, intensidade, natureza e tempo de exposição (BRASIL, 1995).

Quando os resíduos, especialmente os RSS, são gerenciados de forma inadequada podem expor as pessoas que trabalham ou tiveram contato a sérios riscos. De acordo com Cussiol (2008), essas pessoas podem estar expostas a risco biológico, físico, químico, ergonômico e também de acidentes.

A Resolução da Diretoria Colegiada da Agência Nacional de Vigilância Sanitária (Anvisa), RDC n 306/04 e a Resolução do Conselho Nacional do Meio Ambiente (Conama) n 358/05 classificam os RSS em cinco grupos: Grupo A (Biológicos); Grupo B (Químicos); Grupo C (Radioativos); Grupo D (Comuns) e Grupo E (Perfurocortantes) (BRASIL, 2004, 2005).

Anualmente, quantidades significativas desses resíduos são geradas em Instituições de Ensino Superior (IES). Di Vitta et al. (2010) destacam a importância da realização do tratamento e armazenamento adequado visando minimizar os efeitos da introdução desses resíduos no ambiente.

Frente a esse contexto, vários estudos em instituições que geram RSS começaram a voltar seus olhares para a questão dos resíduos produzidos em suas delimitações (SILVA; HOPPE, 2005; PUGLIESI; GIL; SCHALCH, 2009), com destaque para os resíduos químicos presentes em muitas dessas instituições devido a sua variabilidade e especificidade (CUNHA, 2001; JARDIM, 1998; MISTURA; HÄRTER; LINCK, 2010; NOLASCO; TAVARES; BENDASSOLLI, 2006; DI VITTA et al., 2010).

Os benefícios obtidos com a minimização da geração de resíduos químicos nessas instituições aliado a utilização de técnicas e procedimentos adequados em seu manejo são atitudes que colaboram com a segurança dos funcionários, técnicos, estudantes, proporcionando, ainda, esse benefício à comunidade ao prevenir a contaminação dos recursos naturais.

Assim, como em vários segmentos da sociedade, as IES têm realizado estudos na área de Gestão Ambiental, com especial atenção em relação aos laboratórios de ensino, pesquisa e extensão, e outros serviços responsáveis pela geração de resíduos nas universidades. Essas instituições apresentam um papel fundamental nesse processo, 
pois, apesar de sua importância em relação à produção de conhecimentos científicos, acabam, também, sendo fontes geradoras de resíduos de diferentes naturezas, muitos deles perigosos (PHILIPPI JR; AGUIAR, 2005).

Este estudo tem como objetivo verificar a forma do manejo interno (segregação, acondicionamento, identificação, armazenamento, coleta, transporte e tratamento) e externo (coleta, transporte, tratamento e destinação final) dos resíduos químicos nos laboratórios e serviços do Campus da Universidade de São Paulo em Ribeirão Preto (BRASIL, 2004, 2005).

\section{METODOLOGIA}

Para realizar a coleta de dados nesta investigação, foi elaborado um instrumento de pesquisa fundamentado nas diretrizes das resoluções n`358/05, do Conama e da RDC n`306/04 da Anvisa (BRASIL, 2004, 2005), além dos princípios e orientações da NBR 10.004/04 (ABNT, 2004) e da Política Nacional de Resíduos Sólidos (PNRS) (BRASIL, 2010).

Neste questionário foram adotados critérios para determinar o conteúdo, tamanho, organização e clareza das questões, consideradas características fundamentais para estimular o informante a responder às questões abordadas (BARROS; LEHFELD, 1986). Buscou-se ainda a imparcialidade e uma organização clara, precisa e de fácil compreensão.

Foi elaborado um cronograma para realizar a visita em cada um dos laboratórios e serviços no referido Campus a fim de convidar os responsáveis por esses locais para participar da pesquisa. Os sujeitos que aceitaram o convite assinaram o Termo de Consentimento Livre e Esclarecido, sendo deixado o questionário para preenchimento em horários que não comprometessem suas atividades. Novas visitas foram realizadas, nas respectivas datas agendadas, para o recolhimento dos questionários.

Os dados foram organizados e analisados segundo as diretrizes da RDC $n^{\circ} 306 / 04$ e da resolução $n^{\circ}$ 358/05 a fim de diagnosticar a forma de manejo interno e externo dos resíduos químicos nessa instituição. 

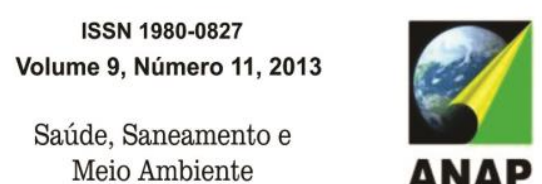

ANAP

A pesquisa de campo só teve início após aprovação Comitê de Ética em Pesquisa da Escola de Enfermagem de Ribeirão Preto da Universidade de São Paulo.

\section{RESULTADOS E DISCUSSÃO}

Do total de 299 laboratórios e serviços do Campus convidados para participar desta pesquisa, ocorreu à participação efetiva de 199 sujeitos, respondendo por um percentual de $66,6 \%$ dos locais da pesquisa.

Segundo os sujeitos, em $80,9 \%$ desses locais havia a geração de resíduos químicos, com uma grande diversidade desse tipo de resíduo, tanto no estado líquido como sólido.

Destaca-se que muitos desses resíduos apresentam características perigosas como inflamabilidade, corrosividade, reatividade, toxicidade e carcinogenicidade. Assim, esses resíduos podem ser responsáveis por expor as pessoas a riscos e causar sérios acidentes quando não manejados adequadamente.

Vale ressaltar que entre os sujeitos que relataram gerar resíduos químicos, 69,4\% informaram que realizavam a segregação no próprio local de sua geração, 13,8\% dos sujeitos realizavam a segregação posteriormente, 6,6\% não realizavam e 8,4\% não tinham conhecimento. Esses dados revelam uma situação preocupante no manejo desses resíduos devido à diversidade de suas características, pois quando não são segregados adequadamente podem gerar problemas nas demais fases do gerenciamento, elevando 0 risco tanto para exposição humana, quanto para o ambiente.

Ocorreu uma grande variação nas respostas referentes ao tipo de acondicionamento usado para os resíduos químicos. A resposta mais frequente foi referente ao armazenamento em recipientes de vidro separadamente (46,9\%). Para esse grupo de resíduos, o fato de haver uma grande variação de forma de acondicionamento, não revela necessariamente haver discordância com as normas vigentes, visto que a Anvisa destaca que para o acondicionamento dos resíduos do Grupo B devem ser observadas as exigências de compatibilidade química dos resíduos com os materiais das embalagens, de forma a evitar possíveis reações químicas (BRASIL, 2004). 
Dessa forma, podem ser utilizados recipientes de vidro, plástico ou metal, contanto que as características dos resíduos sejam compatíveis com o recipiente utilizado. Outro cuidado que deve ser adotado é em relação ao acondicionamento de mais de um tipo de resíduo químico misturado, o que pode causar acidentes, devendo ser analisada a compatibilidade entre os resíduos antes de se acondicionar em um mesmo recipiente mais de um tipo de substância química, segundo as especificações fornecidas pelo fabricante (BRASIL, 2006; UNIVERSIDADE DE SÃO PAULO, 2010a, 2010b).

Quanto à identificação das embalagens utilizadas no acondicionamento, essa era uma prática adotada em 65,8\% dos laboratórios e serviços estudados, enquanto 25,6\% não eram identificadas e $8,6 \%$ dos sujeitos não tinham conhecimento sobre essa fase do manejo.

Dentre as respostas informadas pelos sujeitos quanto a um local exclusivo para armazenamento interno ou temporário, 47,2\% dos sujeitos referiram não ter esse local, 43,7\% afirmaram ter e 9,1\% dos sujeitos não tinham conhecimento.

Em relação à coleta e transporte interno, foi informado que a maioria das pessoas que realizavam a coleta interna era funcionário da própria instituição $(50,8 \%)$, com uma maior utilização de transporte manual $(63,3 \%)$ e realizado diariamente ou semanalmente em $60,1 \%$ dos casos. Ressalta-se que apesar da maioria dos funcionários que realizavam essa etapa do manejo ser do quadro próprio, foi indicado que 36,7\% correspondiam a funcionários terceirizados, fator que dificulta o planejamento de treinamentos, devido à alta rotatividade.

Ao considerar o tratamento interno, foi informado por $28,1 \%$ dos sujeitos que em seus locais de trabalho era realizado o tratamento interno dos resíduos gerados, dado que demonstra um potencial nessa área, sendo necessário investir no treinamento de técnicos e outros profissionais nesses locais visando proporcionar uma otimização no processo e a minimização de acidentes, além do atendimento à legislação. Contudo, os cuidados necessários devem ser adotados ao selecionar os resíduos químicos a serem tratados no próprio local de sua geração, pois, devido às questões de segurança e legislação, alguns tipos desses resíduos devem ser encaminhados a laboratórios específicos e autorizados para o seu tratamento.

O manejo externo dos resíduos químicos (coleta, transporte, tratamento e disposição final), segundo a legislação vigente, é de responsabilidade da instituição 
geradora. A coleta e o transporte externos correspondem às primeiras etapas do manejo externo, responsáveis pela remoção dos resíduos do local de sua geração até a unidade de tratamento ou disposição final, utilizando técnicas que garantam a preservação das condições dos recipientes ou embalagens e a integridade dos funcionários da coleta, da população e do ambiente, e devem estar de acordo com as normas e orientações dos órgãos de limpeza pública municipal (BRASIL, 2004).

A respeito da coleta externa, $34,3 \%$ das respostas dos sujeitos indicaram que os responsáveis correspondiam aos funcionários do Laboratório de Resíduos Químicos do Campus e $42,9 \%$ das respostas indicavam o desconhecimento do tipo de veículo utilizado.

Em relação ao tratamento externo, $29,1 \%$ dos sujeitos indicaram que o tratamento realizado para os resíduos químicos correspondiam à recuperação, 28,3\% informaram que os resíduos eram neutralizados e $21,3 \%$ incinerados. Entre os sujeitos, $20,5 \%$ não tinham conhecimento sobre os tipos de tratamento destinados a esses resíduos e $0,8 \%$ indicaram o Laboratório de Resíduos Químicos do Campus como tratamento, o que na verdade, corresponde ao local no qual é realizado o tratamento e não ao método.

A destinação final inclui a reutilização, reciclagem, compostagem, recuperação e aproveitamento energético. Já a disposição final ambientalmente correta tem o foco centrado nos aterros sanitários (BRASIL, 2010; RODRIGUES, 2012). Quando respondidas as questões referentes à disposição final, $66,7 \%$ das respostas correspondiam ao desconhecimento dessa fase do manejo.

\section{CONCLUSÕES}

A legislação brasileira comtempla os resíduos químicos na definição dos resíduos de serviços de saúde, e estabelece que todo gerador desse tipo de resíduo é responsável por todas as suas fases de manejo e pela sua disposição final ambientalmente correta.

No Campus foram observadas várias técnicas e procedimentos que estavam sendo realizados dentro das especificações das normas brasileiras vigentes, porém, ainda 
ocorria a utilização de práticas não condizentes com as exigências legais. Dessa forma, destaca-se a importância da elaboração e acompanhamento de um Plano de Gerenciamento de Resíduos nas Instituições de Ensino Superior que possuam laboratórios e serviços responsáveis por gerar esses tipos de resíduos.

Ao responder questões referentes às etapas de manejo externo, os sujeitos demonstraram um alto desconhecimento sobre o que é feito com esses resíduos depois que saem do local em que foram gerados. Os sujeitos apresentaram em suas respostas, não só o desconhecimento, mas a compreensão errônea de alguns conceitos, confundindo os termos apropriados às diferentes fases do manejo externo. $O$ desconhecimento sobre procedimentos adequados, que devem ser adotados pode gerar sérios problemas para o gerenciamento desses resíduos, não colaborando para a qualidade ambiental e de saúde do local do estudo.

Outro fator importante corresponde à elaboração de protocolos com orientações básicas sobre como realizar as diferentes fases de manejo dos resíduos químicos que são gerados com maior frequência nessas instituições. Esse material pode ser utilizado em treinamentos com os funcionários e também podem ser disponibilizados para que os técnicos e professores tenham os mesmos disponíveis para consulta em seus laboratórios e serviços.

\section{Agradecimentos}

Esta pesquisa teve o apoio financeiro do Conselho Nacional do Desenvolvimento Científico e Tecnológico (CNPq).

\section{REFERÊNCIAS}

ABNT. NBR 10.004: Resíduos sólidos - Classificação. Rio de Janeiro: 2004. 71 p.

BARROS, Adil Jesus Paes Barros; LEHFELD, Neide Aparecida Souza. Fundamentos de metodologia: um guia para a inicialização científica. São Paulo: McGraw-Hill, 1986. 122 p. 
BUENO, Diana Cruz Fagundes; LEAL, Antonio Cezar. Geração e Destinação dos Resíduos Sólidos Domiciliares no Pontal do Paranapanema / São Paulo - Brasil. Fórum Ambiental da Alta Paulista, v. 8, n. 12 p. 159-176, 2012.

BRASIL. Ministério do Trabalho e Emprego. Secretaria de Segurança e Saúde no Trabalho. Portaria $\mathrm{n}-25$, de 29 de dezembro de 1994. Aprova o texto da Norma Regulamentadora no 9 - Risco Ambientais. Diário Oficial da União. Brasília, DF, 15 dez. 1995. Seção I, p. 1987 a 1989.

Agência Nacional de Vigilância Sanitária. Resolução RDC n³06, de 07 de dezembro de 2004. Dispõe sobre o regulamento técnico para o gerenciamento de resíduos de serviços de saúde. Diário Oficial da União. Brasília, DF, 10 dez. 2004.

- Conselho Nacional do Meio Ambiente. Resolução $n^{\circ}$ 358. Dispõe sobre o tratamento e a disposição final dos resíduos de saúde e dá outras providências. Diário Oficial da União. Brasília, DF, 04 maio 2005.

- Ministério da Saúde. Manual de gerenciamento de resíduos de serviço de saúde. Brasília, DF: Ministério da Saúde, 2006. 182p.

Lei no 12.305, de 02 de agosto de 2010. Institui a Política Nacional de Resíduos Sólidos; altera a Lei ํํ 9.605, de 12 de fevereiro de 1998; e dá outras providencias. Diário Oficial da União. Brasília, DF, 03 ago. 2010.

CUNHA, Carlos Jorge. O Programa de Gerenciamento dos Resíduos Laboratoriais do Departamento de Química da UFPR. Química Nova, São Paulo, v. 24, n. 3, p. 424-427, 2001.

CUSSIOL, Noil Amorim Menezes. Manual de Gerenciamento de Resíduos de Serviços de Saúde. Fundação Estadual do Meio Ambiente. Belo Horizonte: Feam, 2008. 88 p.

DI VITTA, Patrícia Busko et al. Sistemas de gestão de resíduos do Instituto de Química da Universidade de São Paulo. In: DE CONTO, Suzana Maria. Gestão de Resíduos em Universidades. Caxias do Sul, RS: Educs, 2010. cap. 12, p. 271-305.

JARDIM, Wilson Figueiredo. Gerenciamento de resíduos químicos em laboratórios de ensino e pesquisa. Revista Química Nova, São Paulo, v. 21, n. 5, p. 671-673, set./out. 1998.

MISTURA, Clóvia Marozzin; VANIEL, Ana Paula Härter; LINCK, Mara Regina. Gerenciamento de Resíduos dos Laboratórios de Ensino de Química da Universidade de Passo Fundo, RS. Revista CIATEC, v. 2, n. 1, p. 54-64, 2010. 
NOLASCO, Felipe Rufine; TAVARES, Glauco Arnold; BENDASSOLLI, José Albertino. Implantação de Programas de Gerenciamento de Resíduos Químicos Laboratoriais em Universidades: Análise Crítica e Recomendações. Engenharia Sanitária e Ambiental, v. 11, n. 2, p. 118-124, abr./jun. 2006.

PHILIPPI JR, Arlindo; AGUIAR. Alexandre Oliveira. O. Resíduos sólidos: características e gerenciamento. In: PHILIPPI JR, Arlindo. Saneamento, saúde e ambiente: fundamentos para um desenvolvimento sustentável. São Paulo: Manole, 2005. cap. 8, p. 267-321.

PUGLIESI, Erica; GIL, Tatiana Novis Lopes; SCHALCH, Valdir. Caracterização qualitativa e quantitativa dos resíduos de serviço de saúde gerados em hospital de médio porte no município de São Carlos, SP. Revista Minerva, São Carlos, v. 6, n. 2, p. 213-217, maio/ago. 2009.

RODRIGUES, Fernando Altino. Resíduos Perigosos. In: JARDIM, Arnaldo; YOSHIDA, Consuelo; MACHADO FILHO, José Valverde. Política Nacional, Gestão e Gerenciamento de Resíduos Sólidos. São Paulo: Manole, 2012. cap. 21p. 501-514.

SILVA, Carlos Ernando; HOPPE, Alessandro Eduardo. Diagnóstico dos Resíduos de Serviços de Saúde no interior do Rio Grande do Sul. Engenharia Sanitária e Ambiental, v. 10, n. 2, p. 146-151, abr./jun. 2005.

TAKAYANAGUI, Angela Maria Magosso. Gerenciamento de resíduos de serviços de saúde. In: PHILIPPI JR, Arlindo. Saneamento, saúde e ambiente: fundamentos para um desenvolvimento sustentável. São Paulo: Manole, 2005. cap. 9, p. 323-374.

UNIVERSIDADE DE SÃO PAULO. Gerenciamento de resíduos químicos - Normas e procedimentos. São Paulo: Universidade de São Paulo - Prefeitura do Campus Administrativo de Ribeirão Preto - Laboratório de Resíduos Químicos. Disponível em: < http://www.pcarp.usp.br/pages//rq/pdf/normas gerenciamento.pdf >. Acesso em: 22 mai. 2010a. 34 p.

Cartilha de orientação para descarte de resíduo no sistema FMUSP-HC. São Paulo: Hospital das Clínicas - FMUSP. Disponível em: < http://medicina.fm.usp.br/gdc/docs/grss 2 cartilha.pdf >. Acesso em: 22 mai. 2010 b. 40 p. 